\title{
Hadronic Production of Thermal Photons
}

\author{
Simon Turbide ${ }^{1}$, Ralf Rapp ${ }^{2}$, and Charles Gale ${ }^{1}$ \\ 1 Department of Physics, McGill University, 3600 University Street, Montreal, Canada H3A 2 T8 \\ 2 NORDITA, Blegdamsvej 17, DK-2100 Copenhagen, Denmark
}

(Dated: October 25, 2018)

\begin{abstract}
We study the thermal emission of photons from hot and dense strongly interacting hadronic matter at temperatures close to the expected phase transition to the Quark-Gluon Plasma (QGP). Earlier calculations of photon radiation from ensembles of interacting mesons are re-examined with additional constraints, including new production channels as well as an assessment of hadronic form factor effects. Whereas strangeness-induced photon yields turn out to be moderate, the hitherto not considered $t$-channel exchange of $\omega$-mesons is found to contribute appreciably for photon energies above $\sim 1.5 \mathrm{GeV}$. The role of baryonic effects is assessed using existing many-body calculations of lepton pair production. We argue that our combined results constitute a rather realistic emission rate, appropriate for applications in relativistic heavy-ion collisions. Supplemented with recent evaluations of QGP emission, and an estimate for primordial (hard) production, we compute photon spectra at SPS, RHIC and LHC energies.
\end{abstract}

\section{INTRODUCTION}

Electromagnetic radiation (real and virtual photons) from central high-energy collisions of heavy nuclei $(A-A)$ has the potential to directly probe the high temperature and high density phases of these reactions. This is so because photons and dileptons suffer little final state interaction, and also because their emission rate is a rapidly varying function of local intensive variables. It is therefore hoped that electromagnetic (e.m.) observables reveal novel features of strongly interacting systems under extreme conditions, including the quark-gluon plasma (QGP) [1], which is but one facet of a rich many-body problem.

More than a decade of careful experiments and of their theoretical interpretation has made clear that a proof of the existence of the QGP requires analyses of many complementary measurements. This, in particular, applies to thermal production of photons and dileptons, since their emissivity is encoded in the same theoretical quantity, i.e. the e.m. current-current correlation function in strong-interaction matter [2].

In this article we focus on the study of real photon emission (see Ref. [3] for recent reviews). An identification of the QGP component in the spectra requires a reliable assessment of competing sources, most notably primordial production in hard nucleon-nucleon $(N-N)$ collisions [4], as well as the later hadronic emission. Owing to the vanishing invariant mass of real photons, all three sources generate essentially smooth spectra, measured as a function of transverse momentum, $q_{t}$. The key properties are then their strength together with a typical spectral slope. For thermal radiation the latter is governed by temperature (as well as collective matter expansion), whereas the overall normalisation is set by a combination of microscopic production mechanisms and the space-time volume of the interacting matter. Naively, one expects QGP radiation (from large temperatures and small volumes) to dominate at large $q_{t}$, and hadron gas (HG) emission at small $q_{t}$. In practice, i.e. within a heavy-ion environment, both ends of the thermal spectra are masked: there is a large background at low $q_{t}$, and the primordial component from hard $N-N$ collisions feeds the high $q_{t}$. The primordial yield in $A-A$ collisions is expected to be modified (as compared to the free $N-N$ case) by nuclear transverse-momentum $\left(k_{T^{-}}\right)$broadening [5, 6] and shadowing [7]. In general, the strength of thermal emissivities in both hadron gas and QGP can also be substantially affected by microscopic in-medium effects. Furthermore, collective (transverse) expansion induces a spectral hardening being more pronounced in the later (hadronic) phases.

Most of the above issues have been addressed in various forms before, e.g. within hydrodynamic simulations [8, 9, 10], transport/cascade models [11, 12, 13] or simple fireball models [14, 15] (where consistency with dilepton observables has been emphasised). In the present paper we are mostly concerned with hadron gas emission, improving previous analyses in several respects. $\pi \rho a_{1}$-meson gas rates are revisited and extended, including strangeness reactions, heavy resonances as well as $t$-channel exchange of the $\omega(782)$. The effects of baryons - which are known to be of prime importance in the dilepton context [16] (as recently further supported by measurements at lower SPS energy [17]) - are extracted based on the same spectral densities that successfully describe the dilepton data, thus ensuring consistency. This also holds for our modelling of the space-time evolution of heavy-ion reactions, which, in particular, accounts for effects of chemical off-equilibrium in the hadronic phase. Finally, for comparison with data from SPS (and for predictions at RHIC and LHC), hadronic emission is supplemented with an estimate of hard (primordial) production, an assessment of the Cronin effect, as well as QGP radiation (complete to leading order) within a schematic evolution of the collision dynamics.

The article is organised as follows. In Sec. III we briefly recall the definition of emission rates, followed by the 
evaluation of hadronic photon radiation from (i) a hot meson gas using a massive Yang-Mills (MYM) approach, extended to the $S U(3)$ (strangeness) sector, with a quantitative assessment of hadronic form factors; (ii) a baryonrich environment, as well as, (iii) additional meson sources beyond the MYM framework. The total hadronic rate is then compared with a recent leading-order QGP result. In Sec. III we employ a simple dynamical model to compare integrated yields with experimental measurements at SPS, and to provide predictions for RHIC and LHC. Finally, Sec. IV contains a summary and conclusions.

\section{THERMAL PHOTON RATES FROM HADRONIC MATTER}

\section{A. Definitions}

In this work we will adopt two different approaches to calculate thermal photon production from hot and dense matter, taking advantage of the respective strengths of each one.

Within the thermal field theory framework, the differential photon emission rate from an equilibrated system can be written in compact form as [2]

$$
\frac{d R_{\gamma}}{d^{4} q}=P_{\mu \nu} W^{\mu \nu}
$$

where

$$
P_{\mu \nu}=4 \pi \alpha \int \frac{d^{3} p}{(2 \pi)^{3} 2 p_{0}} \sum_{\lambda} \varepsilon_{\mu}^{*}(\lambda, p) \varepsilon_{\nu}(\lambda, p) \delta^{(4)}(p-q)
$$

denotes the (polarisation-summed) photon tensor to lowest order in the e.m. coupling $\alpha$. The key quantity is the hadronic tensor $W^{\mu \nu}\left(q ; \mu_{b}, T\right)$, which contains the effects of strong interactions to all orders and is directly related to the imaginary part of the in-medium photon self-energy (or e.m. current-current correlation function), $\Pi_{\mathrm{em}}$, via

$$
W^{\mu \nu}=(-2) f^{B}\left(q_{0} ; T\right) \operatorname{Im} \Pi_{\mathrm{em}}^{\mu \nu}
$$

with $f^{B}\left(q_{0} ; T\right)=1 /\left(\exp \left[q_{0} / T\right]-1\right)$ the Bose distribution function (note the similarity of Eq. (10) to the dilepton rate, which is obtained upon replacing $P_{\mu \nu}$ with the lepton tensor $\left.L_{\mu \nu}\right)$. The photon rate can therefore be cast into the form

$$
q_{0} \frac{d R_{\gamma}}{d^{3} q}=-\frac{\alpha}{\pi^{2}} f^{B}\left(q_{0} ; T\right) \operatorname{Im} \Pi_{\mathrm{em}}^{T}\left(q_{0}=q ; T\right)
$$

Invoking the vector dominance model (VDM) [18], $\Pi_{\mathrm{em}}$ can be directly related to in-medium vector-meson spectral functions (see below), which makes Eq. (4) particularly suitable for non-perturbative model calculations at low and moderate energies and momenta.

Alternatively, the photon emission can also be computed from relativistic kinetic theory. For a given reaction $1+2 \rightarrow 3+\gamma$, the pertinent rate becomes

$$
q_{0} \frac{d R_{\gamma}}{d^{3} q}=\int \frac{d^{3} p_{1}}{2(2 \pi)^{3} E_{1}} \frac{d^{3} p_{2}}{2(2 \pi)^{3} E_{2}} \frac{d^{3} p_{3}}{2(2 \pi)^{3} E_{3}}(2 \pi)^{4} \delta^{(4)}\left(p_{1}+p_{2} \rightarrow p_{3}+q\right)|\mathcal{M}|^{2} \frac{f\left(E_{1}\right) f\left(E_{2}\right)\left[1 \pm f\left(E_{3}\right)\right]}{2(2 \pi)^{3}}
$$

where $\mathcal{M}$ is the invariant scattering matrix element. For photon self-energies of no more than two-loop order, its imaginary part reduces to tree level diagrams, in which case it is usually more convenient to find thermal rates with the kinetic formula, Eq. (5). The perturbative character of this approach thus makes it advantageous over Eq. (4) at moderate and high energies and momenta.

\section{B. Meson Gas and Hadronic Form Factors}

To describe photon-producing reactions in a gas consisting of light pseudo-scalar, vector and axial vector mesons $\left(\pi, K, \rho, K^{*}, a_{1}\right)$ we employ the massive Yang-Mills (MYM) approach, which is capable of yielding adequate hadronic phenomenology at tree level with a rather limited set of adjustable parameters. Vector and axial vector fields are 
implemented into an effective nonlinear $\sigma$-model Lagrangian as massive gauge fields of the chiral $\mathrm{U}(3)_{L} \times \mathrm{U}(3)_{R}$ symmetry [19, 20]:

$$
\begin{aligned}
\mathcal{L}= & \frac{1}{8} F_{\pi}^{2} \operatorname{Tr} D_{\mu} U D^{\mu} U^{\dagger}+\frac{1}{8} F_{\pi}^{2} \operatorname{Tr} M\left(U+U^{\dagger}-2\right) \\
& -\frac{1}{2} \operatorname{Tr}\left(F_{\mu \nu}^{L} F^{L^{\mu \nu}}+F_{\mu \nu}^{R} F^{R^{\mu \nu}}\right)+m_{0}^{2} \operatorname{Tr}\left(A_{\mu}^{L} A^{L^{\mu \nu}}+A_{\mu}^{R} A^{R^{\mu}}\right)+\gamma \operatorname{Tr} F_{\mu \nu}^{L} U F^{R \mu \nu} U^{\dagger} \\
& -i \xi \operatorname{Tr}\left(D_{\mu} U D_{\nu} U^{\dagger} F^{L \mu \nu}+D_{\mu} U^{\dagger} D_{\nu} U F^{R \mu \nu}\right) .
\end{aligned}
$$

In the above,

$$
\begin{aligned}
& U=\exp \left(\frac{2 i}{F_{\pi}} \sum_{i} \frac{\phi_{i} \lambda_{i}}{\sqrt{2}}\right)=\exp \left(\frac{2 i}{F_{\pi}} \phi\right), \\
& A_{\mu}^{L}=\frac{1}{2}\left(V_{\mu}+A_{\mu}\right), \\
& A_{\mu}^{R}=\frac{1}{2}\left(V_{\mu}-A_{\mu}\right), \\
& F_{\mu \nu}^{L, R}=\partial_{\mu} A_{\nu}^{L, R}-\partial_{\nu} A_{\mu}^{L, R}-i g_{0}\left[A_{\mu}^{L, R}, A_{\nu}^{L, R}\right], \\
& D_{\mu} U=\partial_{\mu} U-i g_{0} A_{\mu}^{L} U+i g_{0} U A_{\mu}^{R}, \\
& M=\frac{2}{3}\left[m_{K}^{2}+\frac{1}{2} m_{\pi}^{2}\right]-\frac{2}{\sqrt{3}}\left(m_{K}^{2}-m_{\pi}^{2}\right) \lambda_{8} .
\end{aligned}
$$

Note that $F_{\pi}=135 \mathrm{MeV}$ and that $\lambda_{i}$ is a Gell-Mann matrix. $\phi, V_{\mu}$ and $A_{\mu}$ are, respectively, the pseudo-scalar, vector and axial vector meson matrices. Note that this form of the interaction enables a coherent treatment of the strange and nonstrange fields.

Let us first concentrate on the nonstrange sector $\left(\pi \rho a_{1}\right)$. Following Ref. [20], the four unspecified parameters in the Lagrangian can be inferred from the masses and widths of the $\rho$ and $a_{1}$, allowing for two possible solutions:

$$
\begin{aligned}
& \text { (I) }: \quad \tilde{g}=10.3063, \gamma=0.3405, \xi=0.4473, m_{0}=0.6253 \mathrm{GeV} \\
& \text { (II) }: \quad \tilde{g}=6.4483, \gamma=-0.2913, \xi=0.0585, m_{0}=0.875 \mathrm{GeV}
\end{aligned}
$$

where $\tilde{g}=g_{0} / \sqrt{1-\gamma}$. In the absence of additional empirical constraints, the use of one set over another is difficult to justify. However, in Ref. [21] it has been suggested to invoke the experimental determination of $D$ - to $S$-wave content in the final state of the $a_{1} \rightarrow \rho \pi$ decay. For the two parameter sets given above one finds

$$
\begin{aligned}
& \text { (I) }: D / S=0.36 \\
& \text { (II) }: D / S=-0.099,
\end{aligned}
$$

to be compared to the experimental value $[22]-0.107 \pm 0.016$. From here on, we therefore employ parameter set II.

We proceed with a systematic evaluation of all processes generating photons based on the interaction vertices contained in Eq. (6). The explicit reactions considered include all possible $s$-, $t$ - and $u$-channel (Born-) graphs for the reactions: $X+Y \rightarrow Z+\gamma, \rho \rightarrow Y+Z+\gamma$ and $K^{*} \rightarrow Y+Z+\gamma$. For $X, Y, Z$ we have each combination of $\rho, \pi, K^{*}, K$ mesons which respect the conservation of charge, isospin, strangeness and G-parity defined for nonstrange mesons. The axial $a_{1}$ meson has been considered as exchange particle only (the $a_{1} \rightarrow \pi \gamma$ decay is automatically incorporated via $s$-channel $\pi \rho$ scattering). Using Eq. (5) the thermal photon production rates are readily obtained from the coherently summed matrix elements in each channel, and convenient parametrisations thereof are given in Appendix A The parametrisations for the nonstrange reaction channels in the present work differ from the ones quoted in Ref. 23], which are based on Ref. 20], in two respects. First, in the latter article the amplitude in two channels, as written, violates the Ward identity [24]. Second, the choice of parameters underlying Ref. 23] yields an $D / S$ ratio which is at variance with the experimental value. This is corrected here, together with amplitudes which have been verified for current conservation. As in Ref. [20], parameter set (II) yields smaller emission rates than set (I) for the (leading) $\pi \rho \rightarrow \pi \gamma$ process at high energies. In addition, our results using set (II) are another $\sim 40 \%$ smaller than the corresponding ones in Ref. [20].

An important element in applying effective hadronic models at moderate and high momentum transfers is the use of hadronic vertex form factors simulating finite-size effects, which are not accounted for in the $\pi \rho a_{1}$ calculations of Ref. [20]. For some of these reactions form factor effects have first been studied in Ref. 25] and found to give a typical net suppression over the bare graphs by an appreciable factor of $\sim 3$ at photon energies around $q_{0} \simeq 2.5 \mathrm{GeV}$. We perform an estimate of their effect in the present context as follows. To be consistent with the procedure adopted before for dilepton production [26], we assume a standard dipole form for each hadronic vertex appearing in the 


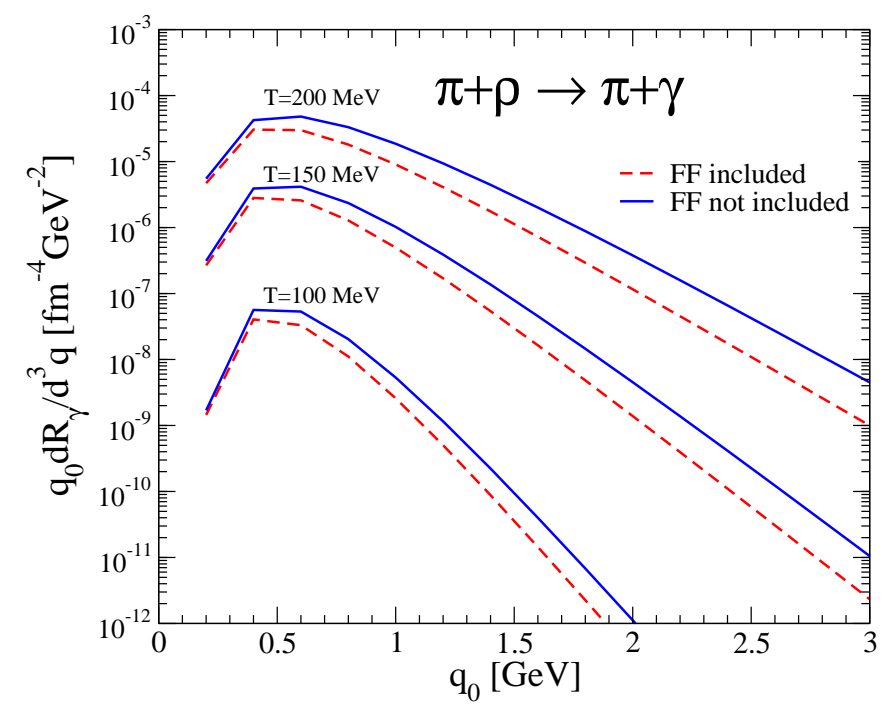

FIG. 1: (Color online) The effect of hadronic form factors on a typical photon-producing reaction in a nonstrange meson gas, $\pi \rho \rightarrow \pi \gamma$, for 3 different temperatures. Solid and dashed lines are without and with the inclusion of $F(\bar{t})^{4}$, respectively.

amplitudes,

$$
F(t)=\left(\frac{2 \Lambda^{2}}{2 \Lambda^{2}-t}\right)^{2}
$$

We then approximate the four-momentum transfer in a given $t$-channel exchange of meson $X$ by its average $\bar{t}$ according to

$$
\left(\frac{1}{m_{X}^{2}-\bar{t}}\right)^{2}\left(\frac{2 \Lambda^{2}}{2 \Lambda^{2}-\bar{t}}\right)^{8}=\frac{1}{4 E^{2}} \int_{0}^{4 E^{2}} \frac{d t\left(2 \Lambda^{2}\right)^{8}}{\left(m_{X}^{2}-t\right)^{2}\left(2 \Lambda^{2}-t\right)^{8}}
$$

The averaging procedure allows to factorize form factors and amplitudes which much facilitates the task of rendering the final expression gauge invariant, since we can then simply multiply the rate parametrisations with $F(\bar{t})^{4}$ employing a typical hadronic scale, $\Lambda=1 \mathrm{GeV}$ [26], for the cutoff parameter. The impact of the form factors is indicated by the difference of solid and dashed curves in Fig. 1 which should still be considered as a conservative estimate of the suppression effect. The reduction of the rate in the $2-3 \mathrm{GeV}$ region amounts to a factor of $\sim 4$, quite in line with the earlier estimates of Ref. 25.

Rather little attention has been paid in the literature so far to the calculation of photon emission rates involving strange particles, mostly because existing analyses have found them to be quantitatively suppressed. In Ref. [27], the channel $K_{1} \rightarrow K \gamma$ was investigated and shown to be appreciable relative to non-strange sources only in a limited kinematical domain. Also, results obtained with $S U(3)$ chiral reduction formulae [28], coupled with an expansion in temperature, suggest that the strangeness contribution is not large. Here, we seek to quantify the latter relative to the $\pi \rho a_{1}$ emissivities within the same effective Lagrangian framework as encoded in the $S U(3)$ extension implicit in Eq. (6). To optimally reproduce the (measured) hadronic phenomenology, we are, however, lead to decouple the nonstrange axial vector meson $\left(a_{1}\right)$ from the strangeness sector. This allows to simultaneously satisfy the (electromagnetic) Ward identities and fix both the strange vector mass, $m_{K^{*}}=895 \mathrm{MeV}$, independent of the $\rho$ mass, and the universal coupling constant as to match the empirical value 22] of the $K^{*}$ width, $\Gamma\left(K^{*} \rightarrow K \pi\right) \simeq 50 \mathrm{MeV}$. The specific channels included are: $\pi K^{*} \rightarrow K \gamma, K \rho \rightarrow K \gamma, \pi K \rightarrow K^{*} \gamma, K K^{*} \rightarrow \pi \gamma, K K \rightarrow \rho \gamma$, and $K^{*} \rightarrow \pi K \gamma$. Again, all possible isospin combinations are accounted for in the rate calculations, which have been parameterized in functional form in Appendix A For a temperature of $200 \mathrm{MeV}$, the leading production channels are shown in Fig. 2] with hadronic form factors implemented following the same procedure, Eq.(11), as before (the last two contributions enumerated above have been omitted, as they represent negligible increments). At all energies of practical relevance $\left(q_{0} \geq 0.5 \mathrm{GeV}\right)$ and 


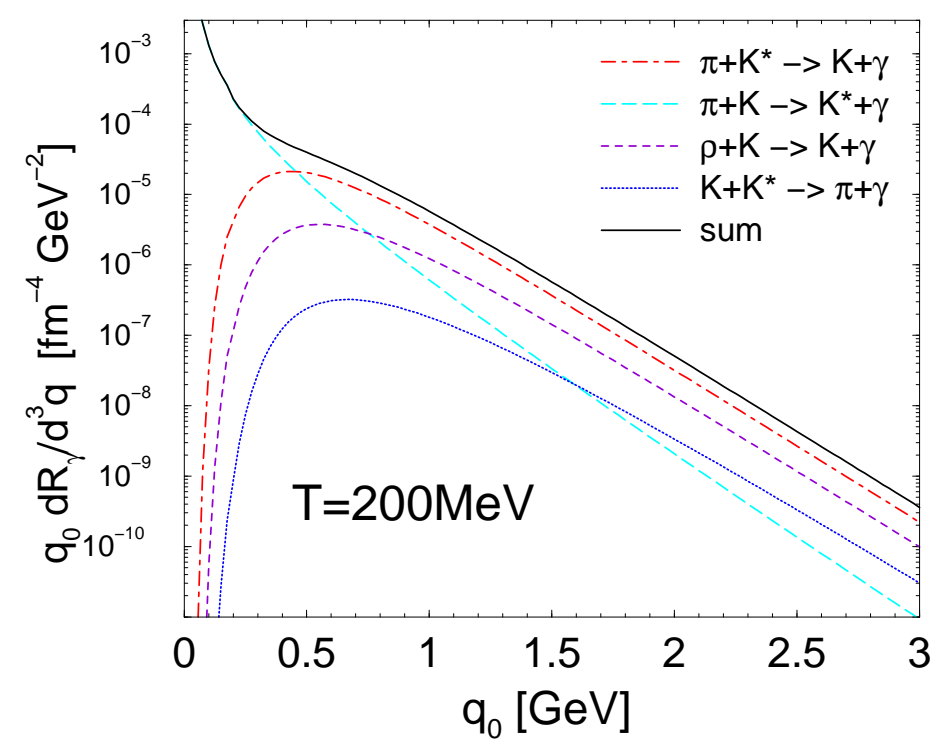

FIG. 2: (Color online) Photon-producing reaction rates involving strange mesons at a temperature $T=200 \mathrm{MeV}$ with form factor effects included.

including form factors, the $K^{*} \pi \rightarrow K \gamma$ reaction, mediated by $t$-channel $\pi$-exchange, turns out to be the main emission source, which is in complete analogy to the $\pi \rho \rightarrow \pi \gamma$ reaction in the nonstrange sector. In line with estimates in [29], the former constitutes $\sim 40 \%$ of the latter around $q_{0}=1 \mathrm{GeV}$, being reduced to about $20 \%$ at $q_{0}=3 \mathrm{GeV}$.

\section{In-Medium Selfenergies with Baryons}

It is important to realize that thermal emission rates of dileptons and photons are intimately connected, both being based on the e.m. current-current correlator, albeit evaluated in distinct kinematical domains, i.e. timelike $\left(M^{2}=q_{0}^{2}-q^{2}>0\right)$ vs. lightlike $\left(M^{2}=0\right)$, respectively. The latter may imply the prevalence of different processes in the corresponding observable, but consistency can and should be tested. In particular, we recall that baryonic sources are very important [16, 30] for understanding the observed excess in low-mass $(M \leq 1 \mathrm{GeV})$ dilepton production in (semi-) central $P b-A u$ collisions at both full $(160 \mathrm{AGeV})$ [31] and lower $(40 \mathrm{AGeV})$ [17] SPS energy. It is therefore mandatory to scrutinize the role of baryons in photon production, especially since most investigations thus far not revealed substantial contributions [32, 33, 34].

We here make use of the hadronic many-body calculations of the in-medium $\rho(770)$ spectral function 35, 36, 37], which, when evaluated for $M^{2} \rightarrow 0$, directly yield pertinent photon emission rates via Eq. (4). Within the vector dominance model (VDM), one has (schematically)

$$
\operatorname{Im} \Pi_{\mathrm{em}}=\sum_{V=\rho, \omega, \phi} \frac{m_{V}^{4}}{g_{V}^{2}} \operatorname{Im} D_{V}
$$

$\left(m_{V}, g_{V}\right.$ and $\operatorname{Im} D_{V}$ : vector-meson masses, coupling constants and spectral functions, respectively). In the following we focus on contributions arising from the $\rho$-meson, which are dominant since $g_{\rho}^{2} / g_{\omega}^{2} \simeq 10$. In addition to in-medium effects in the pion cloud of the $\rho$-meson (encoded in a modified two-pion decay width, $\left.\Sigma_{\rho \pi \pi}\right)$, resonant $\rho-h(h=\pi, K, \rho, N, \Delta, \ldots)$ interactions are incorporated through self-energy expressions of type

$$
\Sigma_{\rho h}^{\mu \nu}\left(q_{0}, \vec{q} ; T\right)=\int \frac{d^{3} p}{(2 \pi)^{3}} \frac{1}{2 \omega_{h}(p)}\left[f^{h}\left(\omega_{h}(p)\right)-f^{\rho h}\left(\omega_{h}(p)+q_{0}\right)\right] \mathcal{M}_{\rho h}^{\mu \nu}(p, q)
$$

where the isospin averaged $\rho$ scattering amplitude $\mathcal{M}_{\rho h}$ is integrated over the thermal distribution $f^{h}\left(\omega_{h}(p)\right)=$ $\left[\exp \left(\omega_{h}(p)\right) / T \pm 1\right]^{-1}$ of the corresponding hadron species $h$ with $\omega_{h}(p)=\sqrt{m_{h}^{2}+\vec{p}^{2}}$. The advantage of writing the self-energy in terms of the forward scattering amplitude is that in-medium resonance widths, accounting for higher order effects in temperature and density, are readily implemented without facing problems of double-counting. The 
latter becomes more difficult to keep track of when evaluating higher order topologies in the kinetic-theory approach represented by Eq. (5) [38]. All of the resonances used in constructing the $\rho$ self-energy are enumerated in Refs. [26, [36], which also contains more details on how the interactions are constrained by hadronic phenomenology.

The results from the hadronic many-body approach are compiled in Fig. B] for two temperature-density values characteristic for meson-to-baryon ratios at full CERN-SPS energy (160 AGeV). The solid curve is the net photon
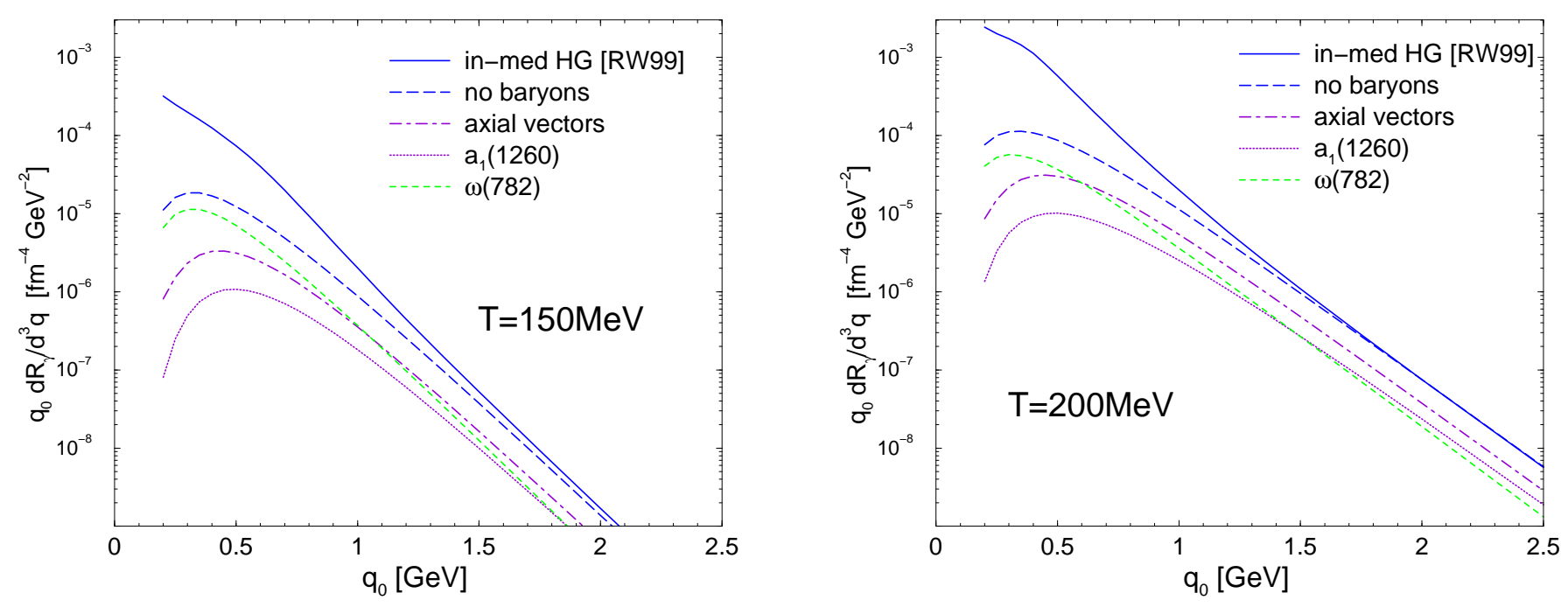

FIG. 3: (Color online) Thermal photon production rate (under conditions resembling CERN-SPS $P b(158 \mathrm{AGeV})+P b$ collisions) in the hadronic many-body approach of Refs. [26, 35, 36] based on an in-medium $\rho$ spectral function. Left panel: for temperature and baryon chemical potential $\left(\mu_{B}, T\right)=(340,150) \mathrm{MeV}$, right panel: $\left(\mu_{B}, T\right)=(220,200) \mathrm{MeV}$.

spectrum obtained by taking the full ( $\rho$-meson) spectral density to the photon point, whereas the long-dashed curve represents the non-baryonic (sub-) component. One observes that the low-energy regime, $q_{0} \lesssim 1 \mathrm{GeV}$, of the photon emissivity is dominated by baryonic effects (quite reminiscent to what has been found for low-mass dileptons). These are mostly due to direct $\rho N$ resonances such as $\Delta(1232), N(1520)$, as well as $\Delta(1232) N^{-1}$ and $N N^{-1}$ excitations in the two-pion cloud of the $\rho$ (which, to leading order in density, correspond to $t$-channel one-pion exchange (OPE) in processes of type $\pi N \rightarrow \gamma N$ ). These contributions should be rather reliable for baryon densities up to at least normal nuclear matter density, $\rho_{0}=0.16 \mathrm{fm}^{-3}$, being constrained by photo-absorption spectra on nucleons and nuclei [37] (including hadronic vertex form factors with rather soft cutoff parameters around $600 \mathrm{MeV}$ ). At comparable baryonic densities this approach yields about a factor of two more photons than the results obtained in Ref. [32, where only nucleonic degrees of freedom where accounted for within the (on-shell) chiral reduction formalism (see Ref. [39] for an update including $\Delta(1232)$ and $N(1520)$ resonances $)$.

Beyond $\sim 1 \mathrm{GeV}$, mesonic (resonance) states become the dominant source of photons in the many-body approach, which includes radiative decays of $\omega(782), h_{1}(1170), a_{1}(1260), f_{1}(1285), \pi(1300), a_{2}(1320), \omega(1420), \omega(1650), K^{*}(892)$ and $K_{1}(1270)$. In particular, the $\omega \rightarrow \pi \gamma$ decay exhibits an appreciable low-energy strength, consistent with the early results of Ref. 25]. Note that all hadronic vertices carry (dipole) form factors with typical cutoff parameters of around $1 \mathrm{GeV}$, as extracted from an optimal fit to measured hadronic and radiative branching ratios within VDM [26]; $t$ channel exchange processes between mesons as discussed in Sec. ПB (e.g. OPE or $a_{1}$-exchange in $\left.\pi \rho \rightarrow \pi \gamma\right)$ are not implicit in the spectral densities leading to the the results of Fig. 3. They are mostly relevant at photon energies beyond $1 \mathrm{GeV}$ and therefore do not significantly figure into bulk (low-mass) dilepton production, the latter being dominated by (transverse) momenta $q_{t} \lesssim M$. As mentioned above, the underlying VDM coupling to the photon exclusively proceeds through the $\rho(770)$ which implies that the strength in the pertinent e.m. correlation function beyond mass/energy scales of $\sim 1 \mathrm{GeV}$ is no longer correctly saturated, as it is restricted to two-pion-type states. The construction of the total emission rate will be discussed in the following Section.

\section{D. $\omega t$-Channel Exchange and Total Rate}

Before combining the various contributions to the thermal photon rate we investigate one more process of potential importance which is not present in the above and, to our knowledge, has not been addressed before. Within the $S U(2)$ flavour symmetry, the $\omega$ is a chiral singlet, but is known to exhibit a large coupling to $\pi \rho$ and thus, via VDM, 


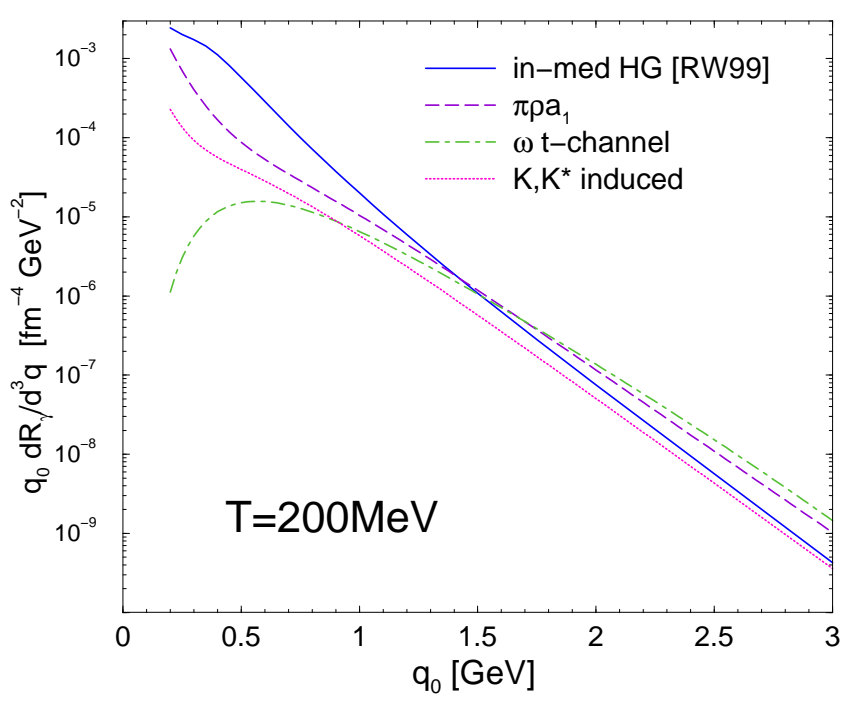

FIG. 4: (Color online) Compilation of thermal photon production rates from hot and dense hadronic matter computed in the present work at temperature $T=200 \mathrm{MeV}$ and baryonic chemical potential $\mu_{B}=220 \mathrm{MeV}$ (translating into total-pion to net-baryon ratio of $\sim 5)$. Dashed and dotted lines correspond to the non-/strange MYM meson gas emissivities of Sec. IIB using the parametrisations given in Appendix $\mathrm{A}$ solid line to the $\rho$ spectral function approach including baryons, and the dashed-dotted line is solely due to $\omega t$-channel exchange in $\pi \rho \rightarrow \pi \gamma$.

to $\pi \gamma$ states. Its $s$-channel decays have indeed been calculated as early as in Ref. [25] (and are included above), but its $t$-channel exchange in the reaction $\pi \rho \rightarrow \pi \gamma$ has not. We here have calculated the pertinent contribution to the thermal emission rate using Eqs. (51) and (11) with the same coupling and form factor type as for the $s$-channel graph [26]. (corresponding to Fig. 33), see below.

In Fig. 4 we summarise our results for the thermal photon emissivities from hadronic matter as evaluated in the preceding Sections. At low energies, $q_{0} \leq 1 \mathrm{GeV}$, the emission rate from the hadronic many-body approach $(\rho$ spectral function) [36], with major contributions from baryonic sources, dominates. Between energies of 1 and $2 \mathrm{GeV}$, meson gas emissivities become competitive and eventually dominate the rates at high energies. Remarkably, the $\omega t$-channel exchange in $\pi \rho \rightarrow \pi \gamma$ is the single most important process beyond energies of $q_{0} \simeq 2 \mathrm{GeV}$. The strangeness component in the production rate does not exceed $10-15 \%$ at any energy.

We finally have to address the question of how to combine the various hadronic sources, computed in two different frameworks (cf. Sec. IA ), into the total emission rate. Two issues arise when simply adding all of the emission rates shown in Fig. 4 double-counting and coherence. The $a_{1} s$-channel graph is present in both $\rho$ spectral function and the MYM framework. We remove it from the former, where it plays a minor role, whereas it induces significant interference effects in the $\pi \rho a_{1}$ complex. If coherence is unimportant, $t$-channel contributions can be evaluated separately. It was verified that this was the case for the $\omega$ exchange, so that the incoherent addition of the $t$-channel contribution is justified.

We believe that it is fair to say that the enumeration of hadronic photons sources given in this Section, together with form factor inclusions, currently represents the most realistic evaluation of the full hadron gas emissivity.

\section{E. Comparison to QGP Emission}

Before turning to applications in heavy-ion reactions, our estimates for hadronic production rates are confronted with the ones from QGP emission, in particular with the simple lowest-order HTL-corrected pQCD result [25],

$$
q_{0} \frac{d R_{\gamma}}{d^{3} q}=\frac{6}{9} \frac{\alpha \alpha_{S}}{2 \pi^{2}} T^{2} \mathrm{e}^{-q_{0} / T} \ln \left(1+\frac{2.912}{4 \pi \alpha_{s}} \frac{q_{0}}{T}\right),
$$

and (a parametrization of) the complete leading-order (in $\alpha \alpha_{\mathrm{s}}$ ) analysis [40], cf. Fig. [5 Clearly, due to the approximations implied by each curve, none of them can be expected to be accurate under conditions arising in practice, i.e. in the phase transition region. Nonetheless, the observation that the complete leading-order QGP calculation (dashed-dotted curve) is similar to the full hadronic result (sum of solid and long-dashed curves) within a factor of 
$\sim 2$ over essentially all (relevant) energies below $3 \mathrm{GeV}$ might not be a mere coincidence. A similar behaviour has been found before for dilepton production rates [36], perhaps suggesting a type of "quark-hadron duality" for e.m. emission close to the expected phase boundary. It would be most valuable to shed further light on this issue from

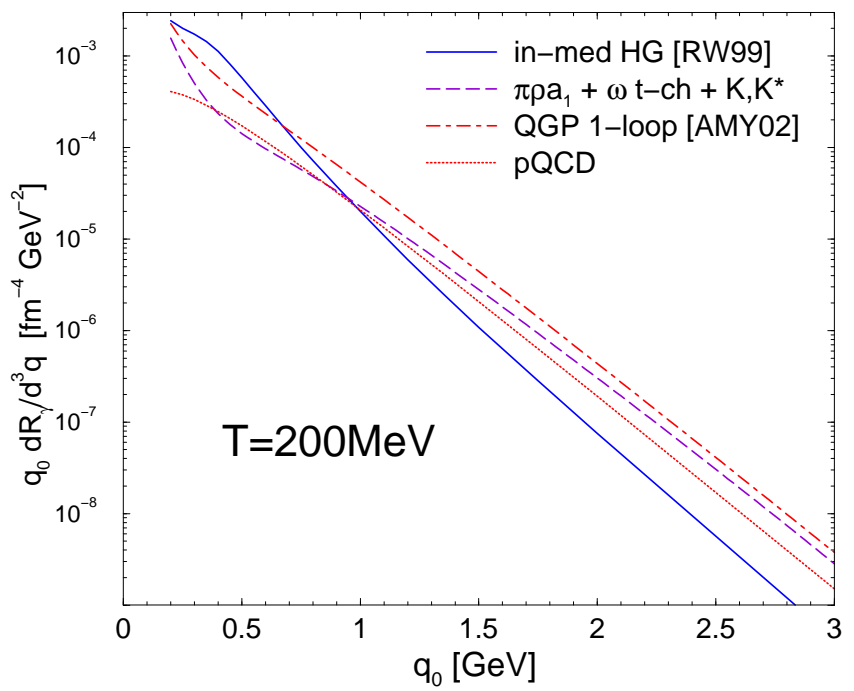

FIG. 5: (Color online) Comparison of HG and QGP photon production rates at $T=200 \mathrm{MeV}$. Solid line: hadronic many-body approach of Refs. [26, 35, 36] (solid curve), dashed line: mesonic contribution including hadronic form factors, dotted line: simple pQCD result [25] according to Eq. (14), dashed-dotted line: complete leading-order QGP emission [40].

first principle lattice calculations, which, at the moment, are only reliable at sufficiently large invariant masses where they are, as expected, close to perturbative results [41].

\section{PHOTON SPECTRA IN ULTRARELATIVISTIC HEAVY-ION COLLISIONS}

\section{A. Hard Photons and Thermal Fireball Evolution}

For a realistic comparison with direct photon spectra as extracted in heavy-ion collisions two further ingredients are required.

First, the thermal rates of the previous sections have to be convoluted over the space-time history of the reaction. Assuming that thermal equilibrium can be established and maintained, hydrodynamic simulations are the method of choice, see e.g. Ref. [10]. Here we employ a more simple fireball model [36, 42], which incorporates essential elements of hydrodynamic calculations. The fireball evolution is started at a "formation" (or thermalization) time $\tau_{0} \leq 1 \mathrm{fm} / \mathrm{c}$, which relates to the initial longitudinal extent of the firecylinder as $\Delta z \simeq \Delta y \tau_{0}$ with $\Delta y \simeq 1.8$ corresponding to the approximate rapidity coverage of a thermal distribution. The subsequent volume expansion, $V_{F B}(\tau)$, is carried through QGP, mixed and hadronic phases until "thermal" freezeout at $T_{f o}=100-120 \mathrm{MeV}$, where hadrons cease to interact. The equations of state (EoS) for QGP and HG are modelled via thermal quasiparticles and a resonance gas (including about 50 species), respectively. Based on the conservation of net baryon-number, $N_{B}$, and total entropy, $S$, one is able to extract the temperature and baryon chemical potential at any given (proper) time, thereby defining a trajectory in the $\mu_{B}-T$ plane. The transition from the QGP to HG phase is placed at "chemical freezeout" points extracted from hadron ratios in experiment [43]. Consequently, in the HG evolution from chemical to thermal freezeout, hadrons stable under strong interactions (pions, kaons, etc.) have to be conserved explicitly by introducing associate chemical potentials $\left(\mu_{\pi}, \mu_{K}\right.$, etc.). This has not been done in previous calculations of thermal photon production [8, 9, 10], and induces a significantly faster cooling in the hadronic phases [29]. In addition, at collider energies (RHIC and LHC), the conservation of the observed antibaryon-to-baryon ratio (which at midrapidities is no longer small) in the hadronic evolution becomes important 44]. An accordingly introduced (effective) chemical potential for antibaryons has been shown to impact the chemistry at later stages appreciably [44] (in particular, it is at the origin of large meson-chemical potentials, again implying faster cooling). For each collision energy, the value of the specific entropy, $S / N_{B}$, is fixed to reproduce observed hadron abundances. The total yield of thermal photons in an $A$ - $A$ collision then 
follows as

$$
q_{0} \frac{d N_{\gamma}^{\text {thermal }}}{d^{3} q}\left(q_{t}\right)=\frac{1}{\Delta y} \int_{y_{\min }}^{y_{\max }} d y \int d \tau V_{F B}(\tau)\left(q_{0} \frac{d R}{d^{3} q}\right)
$$

averaged over a rapidity interval, $\left[y_{\min }, y_{\max }\right]$ according to the experimental coverage $\left(\Delta y=y_{\max }-y_{\min }\right)$. To incorporate transverse expansion in the spectra, in the thermal rest frame isotropic photon momentum distributions are boosted into the laboratory (lab) frame using an average of about $70 \%$ of the time-dependent transverse (surface) expansion velocity at each moment in the fireball evolution.

Second, an additional contribution to direct photon spectra arises from prompt photons in primordial $N-N$ collisions. The minimal baseline for a heavy-ion reaction constitutes the collision-number scaled expectation from proton-nucleon collisions. An accurate description thereof is still a matter of debate 4], so that we here employ empirical scaling relations in $x_{t}=2 q_{t} / \sqrt{s}$, extracted from fits to data in Ref. [45]. For $x_{t} \gtrsim 0.1$, corresponding to fixed target energies $\left(\sqrt{s} \lesssim 50 \mathrm{GeV}\right.$ and photon transverse momenta $q_{t}$ above $2 \mathrm{GeV}$ ), the cross section fit (at midrapidity, $y=0$ ) reads

$$
q_{0} \frac{d^{3} \sigma_{\gamma}^{p p}}{d^{3} q}=575 \frac{(\sqrt{s})^{3.3}}{\left(q_{t}\right)^{9.14}} \frac{\mathrm{pb}}{\mathrm{GeV}^{2}},
$$

whereas for $x_{t} \lesssim 0.1$, corresponding to collider energies $\left(\sqrt{s} \geq 200 \mathrm{GeV}\right.$ and $\left.q_{t} \lesssim 10 \mathrm{GeV}\right)$,

$$
q_{0} \frac{d^{3} \sigma_{\gamma}^{p p}}{d^{3} q}=6495 \frac{\sqrt{s}}{\left(q_{t}\right)^{5}} \frac{\mathrm{pb}}{\mathrm{GeV}^{2}} .
$$

The naive extrapolation to a collision of two nuclei $A$ and $B$ at impact parameter $b$ predicts the prompt photon spectrum to be

$$
\begin{aligned}
q_{0} \frac{d N_{\gamma}^{\text {prompt }}}{d^{3} q}\left(b ; q_{t}, y=0 ; \sqrt{s}\right) & =q_{0} \frac{d^{3} \sigma_{\gamma}^{p p}}{d^{3} q} A B T_{A B}(b) \\
& =q_{0} \frac{d^{3} \sigma_{\gamma}^{p p}}{d^{3} q} \frac{N_{\text {coll }}}{\sigma_{p p}^{i n}}
\end{aligned}
$$

with $T_{A B}$ : nuclear overlap function, $N_{\text {coll }}$ : number of primordial $N-N$ collisions, $\sigma_{p p}^{i n}$ : inelastic $N-N$ cross section (we also supplement Eq. (18) with a smooth cutoff for $q_{0} \leq 2 \mathrm{GeV}$, where the parametrization (16) is no longer reliable). The lack of a consistent microscopic description of photon production in $p-p$ complicates the task to assess nuclear corrections, such as shadowing or intrinsic $k_{T}$ broadening (Cronin effect), see e.g. Ref. [3] for a recent discussion. As a substitute for a more rigorous calculation, we here adopt the following strategy: since the intrinsic $k_{T}$ effects at the $N-N$ level are in principle contained in the parametrization, Eq.(16), the nuclear effect is approximated by fitting an additional (nuclear) $k_{T}$-smearing to $p$ - $A$ data. The latter is modelled by folding the parameterized spectrum over a Gaussian distribution

$$
f\left(k_{T}\right)=\frac{1}{\pi\left\langle\Delta k_{T}^{2}\right\rangle} \mathrm{e}^{-k_{T}^{2} /\left\langle\Delta k_{T}^{2}\right\rangle} .
$$

The result, together with proton-nucleus data on photon production is shown in Fig. 66 The data have been scaled to the $10 \%$ central $\mathrm{Pb}-\mathrm{Pb}$ cross section at $158 \mathrm{AGeV}$ according to the procedure used in Ref. [48]. Fitting the $p$ - $A$ single photon data in this fashion, an adequate reproduction of the experimental measurements emerges with $\left\langle\Delta k_{T}^{2}\right\rangle \simeq 0.1-0.2 \mathrm{GeV}^{2}$.

In principle, there is a third source of photons corresponding to emission after initial nuclear impact, but before the formation time $\tau_{0}$ (the "pre-equilibrium" contribution). It is difficult to assess both theoretically and experimentally; a rough (but uncontrolled) estimate might be had by choosing a somewhat smaller formation time. Note that, in principle, the modelling of those contributions is accessible to ab-initio simulations [49].

\section{B. SPS}

In this section we compute transverse momentum spectra at midrapidities from $10 \%$ central $P b(158 \mathrm{AGeV})+P b$ collisions for which photon spectra have been measured by WA98 [48]. Let us first focus on thermal emission; QGP 
radiation is always calculated with the complete leading-order result [40], and hadronic radiation as the sum of the hadronic many-body 26, 36], $\pi \rho K^{*} K$ gas (within MYM) and $\omega t$-channel exchange contributions with form factors as discussed in Sec. ЩD

In Fig. [7 we display results for a rather standard fireball evolution with initial $z_{0}=1.8 \mathrm{fm}$ (corresponding to $\tau_{0} \simeq$ $1 \mathrm{fm} / \mathrm{c}$ and initial temperature $T_{i}=205 \mathrm{MeV}$ ) and final temperature $T_{f o} \simeq 110 \mathrm{MeV}$ reached after a total lifetime of $\sim 13 \mathrm{fm} / \mathrm{c}$. Due to transverse expansion, the total hadron gas yield outshines QGP emission at all momenta. This is in close reminiscence to the calculations of intermediate dilepton spectra within the same framework [42], where QGP radiation was found to constitute about $30 \%$ of the thermal component that was able to reproduce the excess observed by the NA50 collaboration [50] (see also Ref. [51]). As expected, photons of baryonic origin prevail in the spectrum for $q_{t} \lesssim 1 \mathrm{GeV}$; this region is thus intimately related to the low-mass (and low-transverse momentum) dilepton enhancement observed by CERES/NA45 31]. The same feature has been found [14] when comparing the hadronic many-body contributions to the upper limits in $S(200 \mathrm{AGeV})+A u$ by WA80 [52]. In the right panel of Fig. [7] we illustrate the sensitivity of the hadronic thermal emission to properties of the fireball evolution. When increasing the thermal freezeout time from our (SPS) default value of $106 \mathrm{MeV}$ to $135 \mathrm{MeV}$, the yield at $q_{t} \leq 1 \mathrm{GeV}$ is reduced by up to $30 \%$, whereas it is essentially unchanged beyond $q_{t} \simeq 2 \mathrm{GeV}$, thus reflecting emission close to $T_{c}$. On the contrary, if the additional boost on the photons due to the transverse expansion is neglected, the high-momentum spectrum is reduced appreciably (by a factor of $\sim 3$ already at $q_{t}=2 \mathrm{GeV}$ ), whereas the low-momentum region is only mildly affected.

As is well-known, high-energy (-mass) photon (dilepton) emission is rather sensitive to initial temperatures in heavy-ion reactions, due to the large (negative) exponents in the thermal factors. This is confirmed by our results for the QGP contribution in Fig. 8] when decreasing the formation time from 1 to $0.56 \mathrm{fm} / \mathrm{c}$, the latter implying $T_{i}=250 \mathrm{MeV}$. Another effect that has been ignored in available hydrodynamic calculations so far is associated with corrections to the QGP equation of state. The standard assumption is that of an ideal (massless) gas with an effective number of flavours $N_{f}=2.5$ corresponding to a total degeneracy $d_{Q G P}=\left(10.5 N_{f}+16\right)=42$ (in our default calculations we use $\left.d_{Q G P}=40\right)$. However, lattice gauge theory results [53] indicate $\sim 20 \%$ smaller values than the ideal gas for the thermodynamic state variables in the for SPS energies relevant temperature region $T=1-2 T_{c}$. Implementing such a reduction into the entropy density (which is the relevant quantity for the fireball evolution) by using $d_{Q G P}=32$ yields an increase of the initial temperature from $250 \mathrm{MeV}$ to $270 \mathrm{MeV}$ without decrease in initial volume 29, 54]. The resulting thermal photon spectrum from QGP radiation shows appreciable sensitivity to this modification at high energies, cf. solid vs. dotted curve in Fig. 8. This sensitivity does not persist into the low-energy region $q_{0} \leq 1 \mathrm{GeV}$, and thus does not affect low-mass dilepton production [30].

Let us now turn to a comparison with the recent measurements of WA98 [48]. Our baseline scenario consists of thermal emission (hadronic and QGP) from the expanding fireball with $T_{i}=205 \mathrm{MeV}$, supplemented by prompt

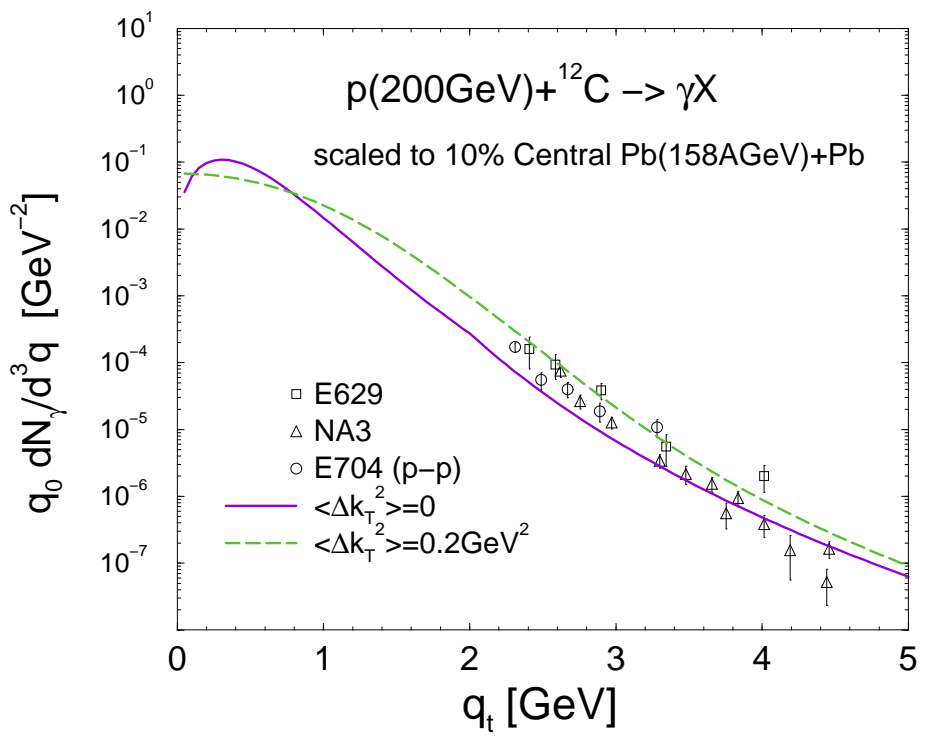

FIG. 6: (Color online) Direct photon data in proton-Carbon collisions, scaled to central $P b-P b$ collisions at SPS energies (see text for details). The curves show the effect of the broadening of the primordial photon spectrum generated by the nuclear medium. The data are from Refs. [46] (E629) and 47] (NA3). 

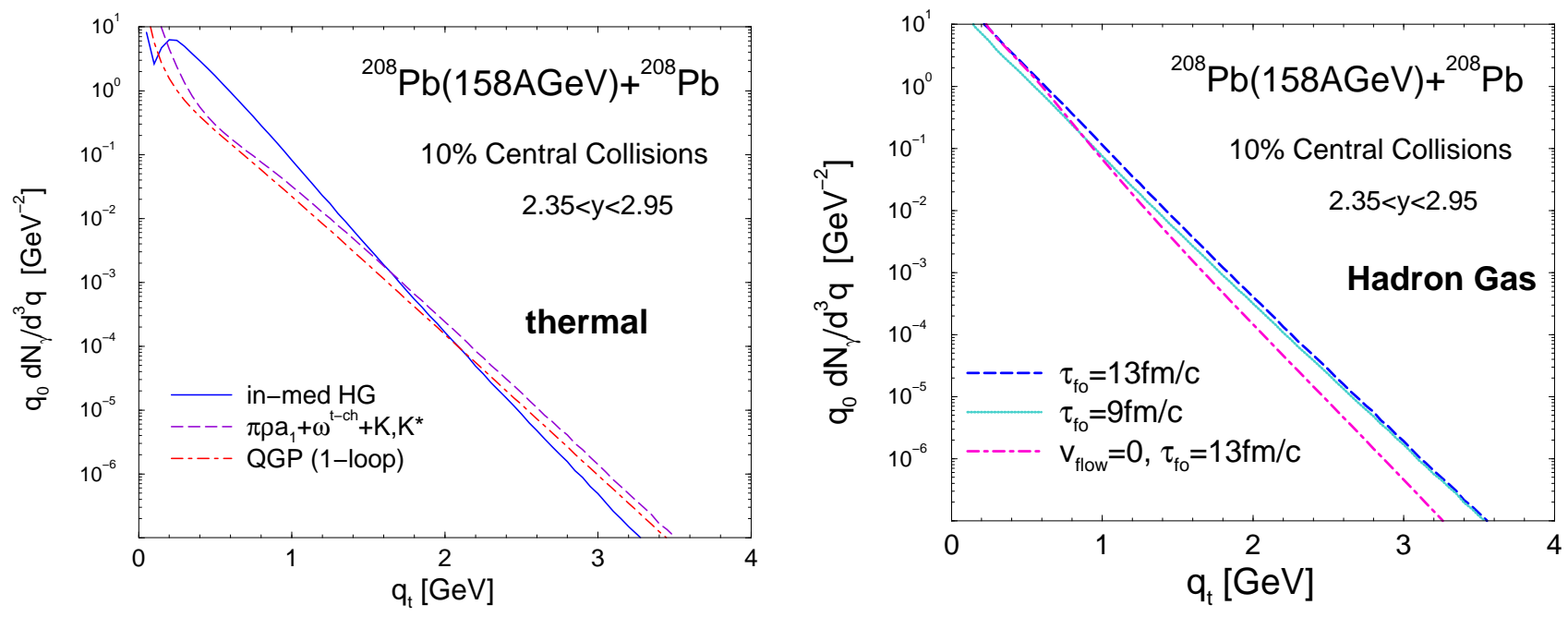

FIG. 7: (Color online) Integrated photon emission from various thermal sources in an expanding fireball model for central $P b+P b$ collisions at SPS. Left panel: hadronic emission $\left(T \leq T_{c}=175 \mathrm{MeV}\right)$ from the meson gas component (dashed line) and the in-medium $\rho$ spectral function (solid line) compared to QGP emission $\left(T_{c} \leq T \leq T_{i}=205 \mathrm{MeV}\right)$ (dashed-dotted line). Right panel: Sensitivity of the total HG yield to thermal freezeout (long-dashed line: $\tau_{f o}=13 \mathrm{fm} / \mathrm{c}$ corresponding to $T_{f o}=106 \mathrm{MeV}$, dotted line: $\tau_{f o}=9 \mathrm{fm} / \mathrm{c}$ corresponding to $T_{f o}=135 \mathrm{MeV}$ ) and transverse flow (dashed-dotted line: $\tau_{f o}=13 \mathrm{fm} / \mathrm{c}$ with no transverse boost of the emission source).

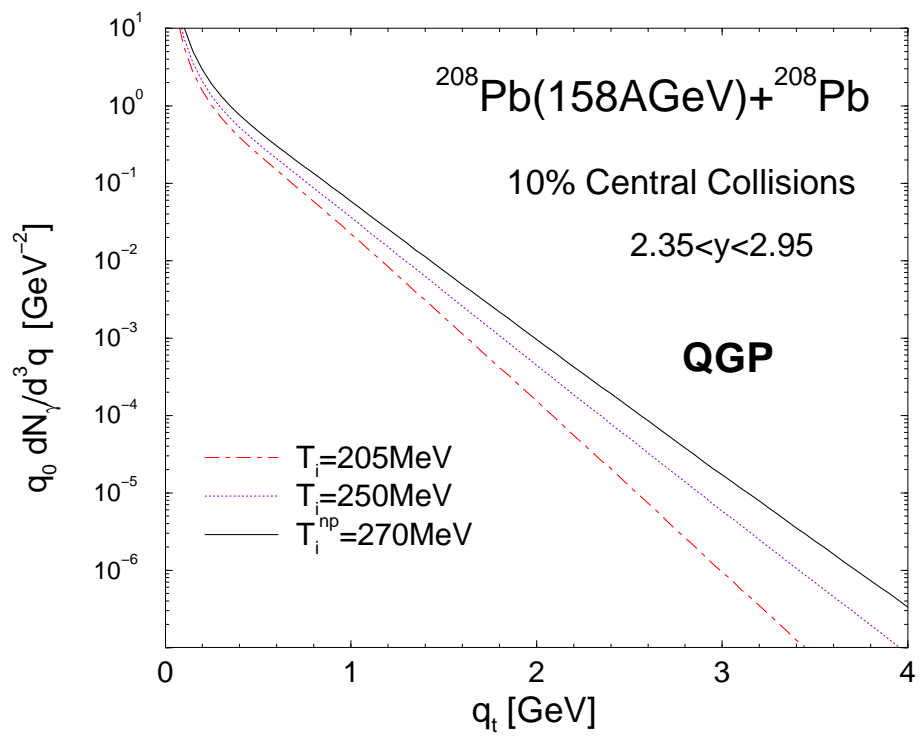

FIG. 8: (Color online) Sensitivity of the QGP photon emission yield within an expanding fireball model for central $P b+P b$ collisions at SPS.

(pQCD) photons from primordial $N-N$ collisions without any nuclear effects, cf. Fig. 9 Up to transverse momenta of about $1.5 \mathrm{GeV}$ the data (upper limits) are essentially saturated by thermal radiation from the hadronic phase. This is gratifying to note since this regime, as discussed above, is directly related to the low-mass dilepton excess observed by CERES/NA45 [17, 31], which can be successfully described within the same approach [36]. Beyond $3 \mathrm{GeV}$, prompt photons dominate, but do not seem to provide enough yield to account for the data. Since the hadron gas emission is essentially fixed and describes well the low-energy regime, three possibilities are left for the origin of discrepancies above $q_{0} \simeq 2 \mathrm{GeV}$ : (i) modifications of the prompt yield, (ii) pre-equilibrium emission, (iii) larger QGP radiation. In the following, cases (i) and (iii) (or a combination thereof) will be investigated.

First, we study the effects of the initial temperature on the photon spectrum. The exercise in the right panel of 


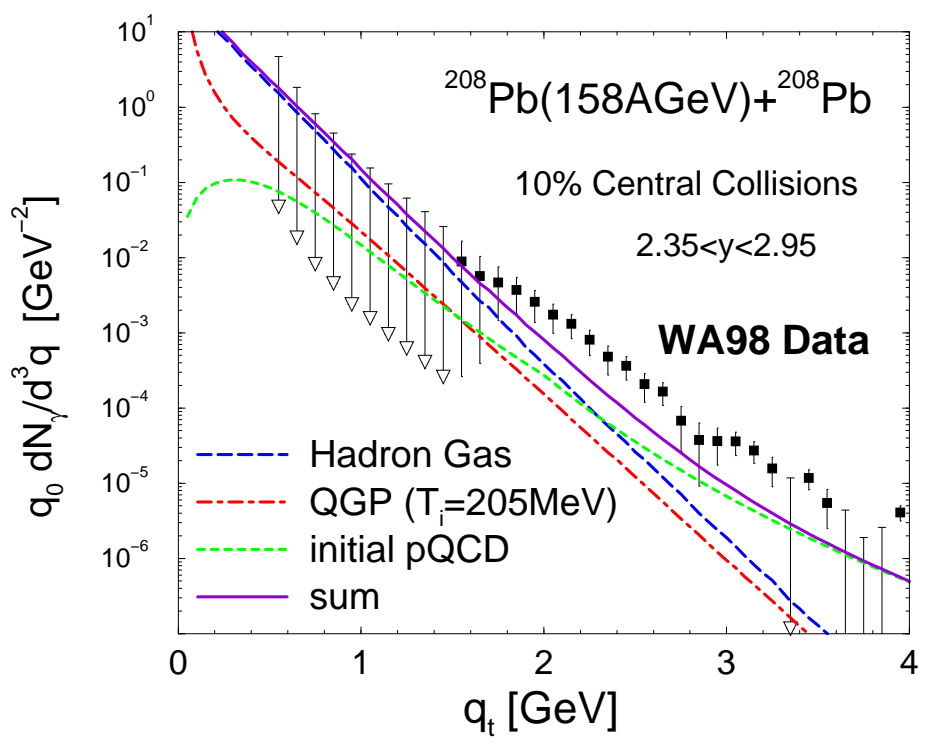

FIG. 9: (Color online) Thermal plus prompt photon spectra compared to data from WA98 48$]$ for central $\mathrm{Pb}+\mathrm{Pb}$ collisions at SPS .

Fig. 7 is repeated adding all sources discussed here, cf. Fig. 10] Clearly, when going to (for SPS conditions) rather short formation times of $\tau_{0} \simeq 0.5 \mathrm{fm} / \mathrm{c}$, coupled with non-perturbative (suppression) effects in the QGP EoS, a rather good reproduction of the entire spectrum can be achieved. This statement agrees with the hydrodynamic analyses of Refs. [9, 10].

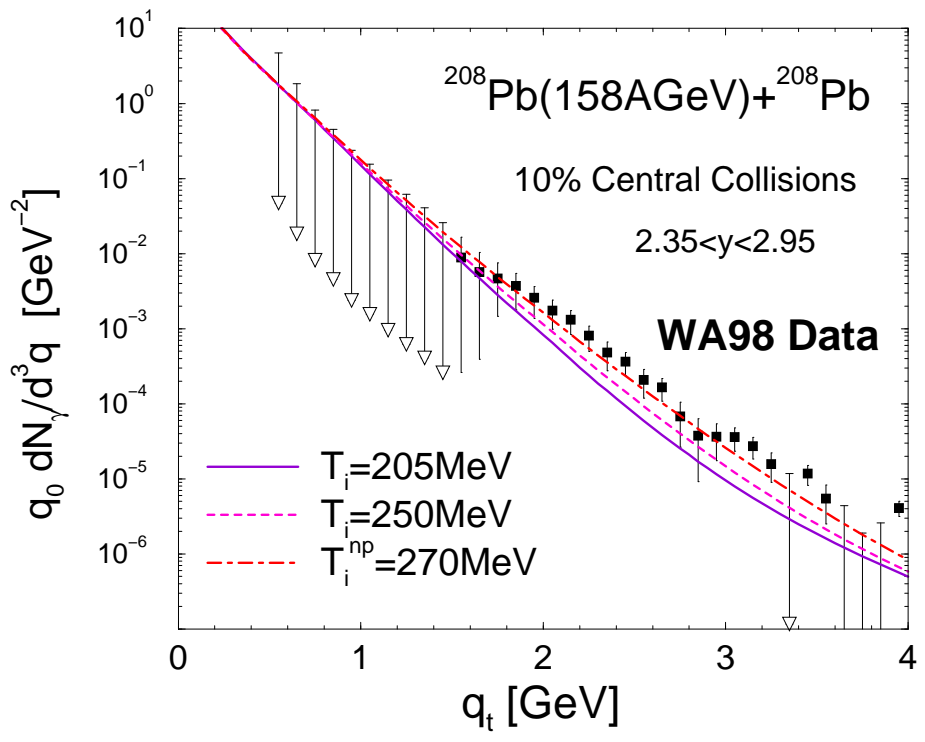

FIG. 10: (Color online) Effects of various initial temperatures on the total photon spectra in $P b+P b$ collisions at SPS, compared to data from WA98 [48].

The second possibility relates to the nuclear Cronin enhancement, which we implement as outlined in the previous Section. The usual assumption to extrapolate nuclear broadening effects on e.g. $\pi^{0}$ or $\gamma$ spectra is that

$$
\left\langle\Delta k_{T}^{2}\right\rangle_{A A}=N\left\langle\Delta k_{T}^{2}\right\rangle_{p A}
$$

with $N=2$ 55. Alternatively, based on a careful analysis of the target $(A)$ dependence in $p$ - $A$ collisions, it has been suggested in Ref. [56] that the Cronin effect is due to no more than one semi-hard collision prior to the hard scattering, 
and therefore saturating as a function of the $N-N$ collision number. In this eventuality, $N \leq 2$. Recalling that, from Fig. [6] $\left\langle\Delta k_{T}^{2}\right\rangle=0.1-0.2 \mathrm{GeV}^{2}$ gives a reasonable description of the $\gamma$ spectra in $p$ - $C,\left\langle\Delta k_{T}^{2}\right\rangle$-values between 0.2 and $0.3 \mathrm{GeV}^{2}$ seem appropriate for central $P b-P b$ collisions. One should also note that the pertinent spectral enhancement in the $q_{t} \simeq 3 \mathrm{GeV}$ region amounts to a factor of around 3, which is quite consistent with the nuclear enhancement in $\pi^{0}$ production observed in the same experiment [57]. In Fig. [1] we have combined the baseline thermal yield $\left(\tau_{0}=1 \mathrm{fm} / \mathrm{c}\right.$, i.e. $T_{i}=205 \mathrm{MeV}$ ) with 3 values for the nuclear $k_{T}$-broadening, i.e. $\left\langle\Delta k_{T}^{2}\right\rangle=0,0.2$ and $0.3 \mathrm{GeV}^{2}$. The thermal plus Cronin-enhanced pQCD spectra provide good description of the WA98 data, even with an initial temperature as low as $T_{i}=205 \mathrm{MeV}$. This constitutes one of the main results of our work: the photon spectrum in nucleus-nucleus collisions at SPS energies is perfectly compatible with "moderate" initial temperatures. It also complements, within a common thermal framework, earlier descriptions of low- and intermediate-mass dilepton spectra 36, 42], as well as $J / \psi$ and $\psi^{\prime}$ production systematics [58], as observed by the CERN-SPS experiments CERES/NA45 [17, 31] and NA50 [50, 59], respectively.

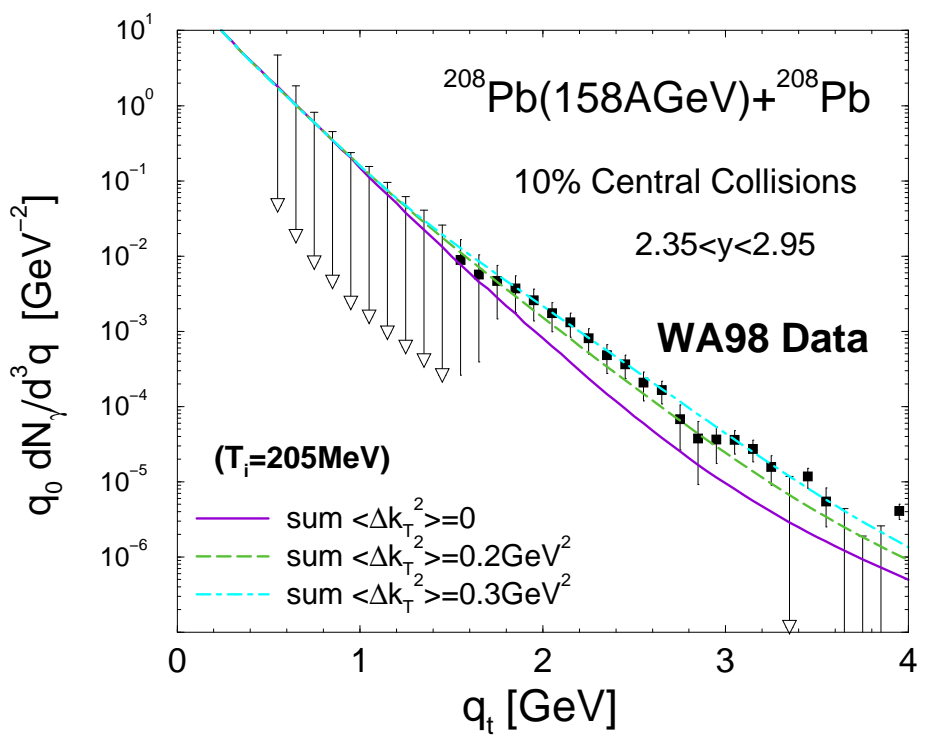

FIG. 11: (Color online) Effects of the nuclear broadening of the primordial photon spectrum on the measured spectrum. All the sources discussed in this paper are included in the space-time evolution.

It is also of interest to quote the values of the transverse momentum where the pQCD yield exceeds the total thermal one; these are $q_{t}=2.55,1.7$ and $1.55 \mathrm{GeV}$, corresponding to $\left\langle\Delta k_{T}^{2}\right\rangle=0,0.2$ and $0.3 \mathrm{GeV}^{2}$, respectively. Again, this compares well with the calculation of intermediate-mass dileptons in Ref. 42], where the Drell-Yan contribution was found to exceed the thermal one at $M_{\mu \mu} \simeq 2 \mathrm{GeV}$ (note that the Cronin effect is expected to be less pronounced for quarks than for gluons, and thus will affect the Drell-Yan process less than prompt photons).

\section{RHIC and LHC}

At collider energies the space-time evolution of the expanding QGP and hadronic fireball is expected to change in several respects. First, higher energies entail larger charged particle multiplicities per unit rapidity, $d N_{c h} / d y$. In central $A u+A u$ collisions at full RHIC energy $(\sqrt{s}=200 \mathrm{AGeV})$ experiments have found [60, 61] about a factor of 2 increase as compared to maximum SPS energy $(\sqrt{s}=17.3 \mathrm{AGeV})$. Extrapolations into the LHC regime $(\sqrt{s}=5500 \mathrm{AGeV})$ suggest another factor of up to $\sim 4$ enhancement over the RHIC results.

Second, the net baryon content at midrapidity decreases, implying small baryon chemical potentials at chemical freezeout, e.g. $\mu_{B} \simeq 25 \mathrm{MeV}$ at RHIC-200. At the same time, the observed production of baryon-antibaryon pairs strongly rises, resulting in total rapidity densities for baryons at RHIC that are quite reminiscent of the situation at SPS energies 62. This observation not only necessitates the explicit conservation of antibaryon-number between chemical and thermal freezeout [44] (see above), but also requires to evaluate baryonic photon sources with the sum of the baryon and antibaryon density (strong and e.m. interactions are CP-invariant).

Third, the transverse expansion (i.e. flow velocity) increases by about $20 \%$ from SPS to RHIC (presumably further at LHC), whereas the total fireball lifetime does not appear to change much. The latter, however, is likely to increase 
at LHC, due the significantly larger system sizes towards thermal freezeout.

All these features are readily implemented [4, 63] into the thermal fireball description employed for SPS energies above. In addition, the primordial pQCD component changes its $x_{t}$-scaling behavior 45] which is accounted for by replacing the parametrization Eq. (16) by Eq. (17). For simplicity we here refrain from introducing a nuclear $k_{T}$ broadening, which is expected to be much less pronounced (and/or compensated by shadowing corrections) at collider energies. First data on high- $p_{t}$ hadron production in $d$ - $A u$ collisions at $\sqrt{s}=200 \mathrm{GeV}[64,65$, 66] indeed indicate only a comparatively small enhancement of around $20-30 \%$ over the spectra measured in $p-p$ collisions.

Our photon predictions for full RHIC energy are summarized in Fig. 12 The thermal component has been evaluated

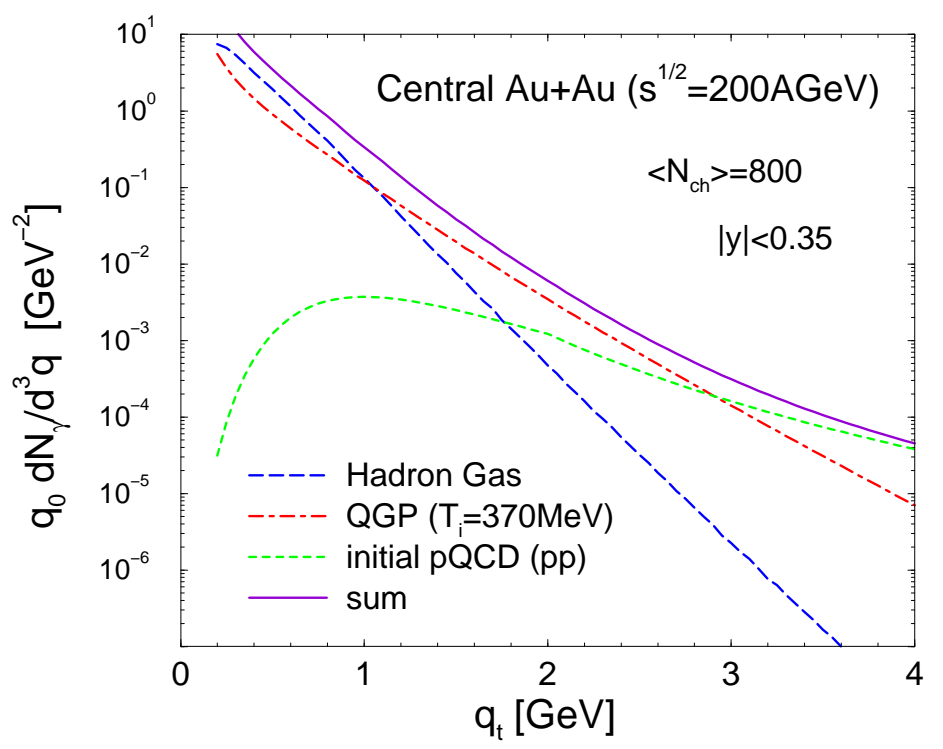

FIG. 12: (Color online) Integrated photon emission spectra from central $A u+A u$ collisions at RHIC. Short-dashed line: pQCD photons from primordial $N-N$ collisions; dashed-dotted line: thermal QGP radiation; long-dashed line: thermal hadron gas emission; solid line: total direct photon yield.

with a typical formation time, $\tau_{0}=1 / 3 \mathrm{fm} / \mathrm{c}$, as used before in dilepton [63] and charmonium [58 applications (it is also consistent with hydrodynamic approaches that correctly reproduce the elliptic flow measurements which are particularly sensitive to the early phases, see Ref. [67] for a recent review). One notes that the spectrum decomposes into essentially 3 regimes: at low energies, $q_{0} \leq 1 \mathrm{GeV}$, the major source are still thermal hadrons, whereas at high energies, $q_{0} \geq 3 \mathrm{GeV}$, prompt pQCD photons dominate. The intermediate region, $1 \leq q_{0} \leq 3 \mathrm{GeV}$, appears to be a promising window to be sensitive for thermal QGP radiation. The latter has been calculated assuming chemically equilibrated quark- and gluon-densities throughout. It is conceivable, however, that the early QGP phases are gluondominated, i.e. with quark fugacities much smaller than one (even the gluon fugacities could be reduced). In this case, on the one hand, the photon emissivities at given temperature are severely suppressed. On the other hand, if most of the total entropy is produced sufficiently early, smaller fugacities imply larger temperatures, thus increasing the photon yield. The interplay of these effects has been studied for dilepton production in Ref. 63], where it has been found that the net effect consists of a slight hardening of the QGP emission spectrum with a pivot point at $M \simeq 3 \mathrm{GeV}$. For photons the situation might be even more favorable due to the participation of gluons in their production (e.g. $g+q \rightarrow g \gamma$, as opposed to leading-order $q \bar{q} \rightarrow e e$ for dileptons).

We finally turn to the LHC, cf. Fig. 13] According to our estimates, assuming a formation time of $0.11 \mathrm{fm} / \mathrm{c}$ (translating into $T_{i} \simeq 850 \mathrm{MeV}$ for $d N_{c h} / d y \simeq 3000$ ), the QGP window extends significantly further in transverse momentum than under RHIC conditions, cf. left panel of Fig. 13] although this feature is sensitive to: (i) the formation time (ii) a possible chemical undersaturation of the QGP, (iii) nuclear effects on the initial pQCD yield. The transition from HG to QGP dominated emission occurs again close to $q_{t}=1 \mathrm{GeV}$. In the right panel of Fig. [13] we illustrate the sensitivity of the thermal spectra with respect to the produced charged particle muliplicity within our schematic fireball evolution model. For simplicity, we assumed the same formation time and expansion parameters for both $N_{c h}=3000$ and 2000 . We then find that the total integrated photon yield (i.e., for transverse momenta above $50 \mathrm{MeV}$ ) scales as $N_{c h}^{\alpha}$ with $\alpha \simeq 1.4$ if the same thermal freezeout temperature $T_{f_{0}} \simeq 90 \mathrm{MeV}$ is imposed. This value for $\alpha$ is somewhat larger than the 1.2 found in hydrodynamic caclulations of Ref. [68], but confirms the deviation from the naive quadratic behavior $(\alpha=2)$. However, the latter is approached (and even exceeded) for the yield at higher transverse momenta; e.g., when integrating over $q_{t}$ with a lower bound of $1 \mathrm{GeV}(2 \mathrm{GeV}), \alpha$ increases to $\sim 1.9(2.3)$. 

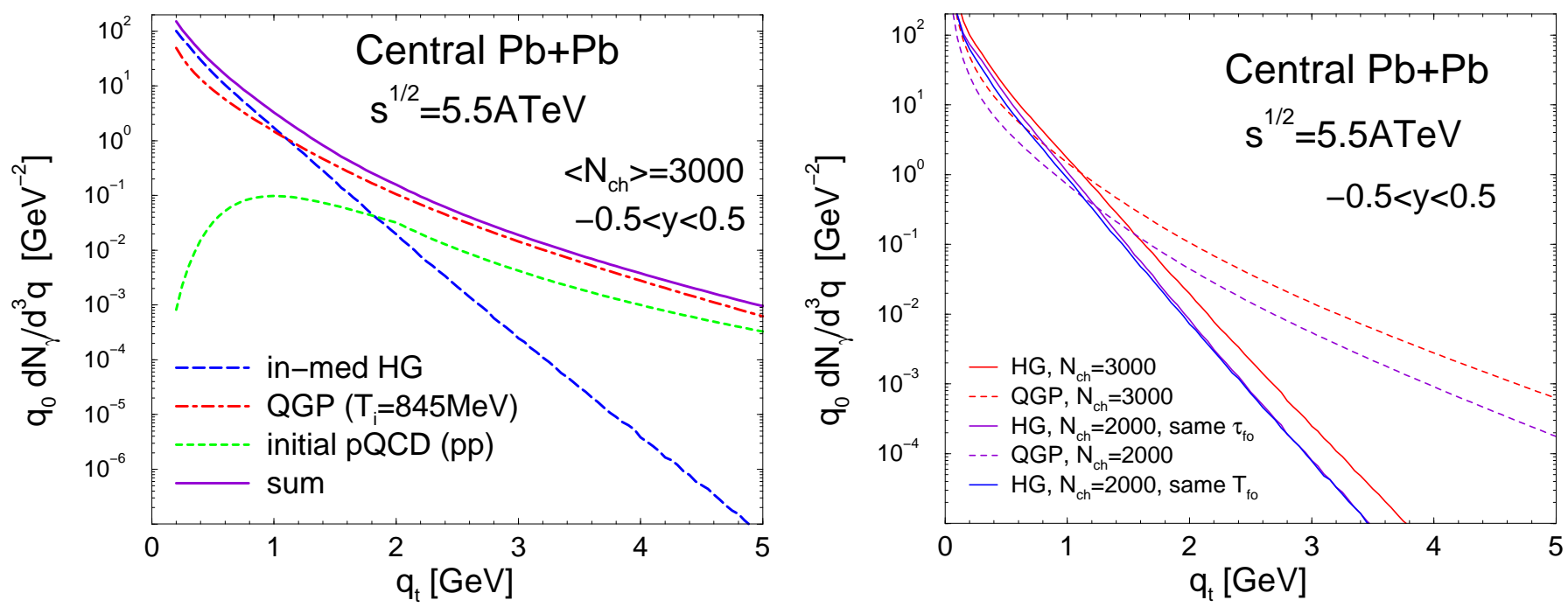

FIG. 13: (Color online) Left panel: integrated photon emission from various thermal sources in central $P b+P b$ collisions at the LHC; line identification as in Fig. 13 Right panel: sensitivity of thermal emission spectra from the hadron gas (solid lines) and the QGP (dahed lines) to the charged particle rapidity density. For HG emission at $N_{c h}=2000$, the upper line corresponds to assuming the same freezeout time $\left(\tau_{f o} \simeq 25 \mathrm{fm} / \mathrm{c}\right)$ as for $N_{c h}=3000$, whereas the lower line corresponds to assuming the same freezeout temperature $\left(T_{\text {fo }} \simeq 90 \mathrm{MeV}\right)$ (implying a $10 \%$ reduced lifetime).

\section{SUMMARY AND CONCLUSIONS}

In the present article we have attempted an improved evaluation of hadronic thermal emission rates for real photons, suitable for realistic applications in relativistic heavy-ion collisions. In what we think is a more complete treatment than has been achieved before, our main findings are:

(i) Revisited meson gas emissivities built upon an effective Lagrangian of the massive Yang-Mills type lead to about $40 \%$ reduced rates in a $\pi \rho a_{1}$ gas as compared to previous analyses. An inclusion of strangeness-bearing channels has revealed that the latter contribute at the $20 \%$ level. A quantitative evaluation of hadronic form factors has been performed throughout, mandatory for applications.

(ii) Photon rates from the baryonic sector have been obtained from a limiting procedure where in-medium $\rho$ spectral densities were carried to the photon point. This procedure makes consistent the real photon analysis with that of low invariant mass dileptons, thereby elucidating the role of baryons in photon emission during nuclear collisions. Their contributions have been shown to be substantial for photon energies $q_{0} \leq 1 \mathrm{GeV}$.

(iii) As the single most important process at high energies we have identified $\omega t$-channel exchange in the $\pi \rho \rightarrow \pi \gamma$ reaction, which had not been considered before.

The total hadronic emissivity has been compared to a recent complete leading-order (in strong and electromagnetic couplings) QCD calculation for the QGP. In the vicinity of the expected phase boundary both rates turned out to be very similar, at all energies of practical relevance.

The net rates have been folded over a fireball evolution of nuclear collisions. This approach, albeit schematic, is consistent with observed hadrochemistry and hydrodynamic expansion characteristics, as well as dilepton and charmonium data measured at SPS energies. Using a comprehensive fit of photon cross sections in $p-p$ and $p$ $\bar{p}$ interactions, an estimate of the Cronin effect (nuclear $k_{T}$-broadening) in $p$ - $A$ collisions was first extracted, then generalised to central $\mathrm{Pb}-\mathrm{Pb}$ collisions to address the WA98 photon measurements at the SPS. Combining the complete set of hadronic rates with QGP emission and our Cronin-effect estimates on the primordial photon component, we are able to reproduce the WA98 data, with moderate values of initial temperature $\left(T_{i} \simeq 200-240 \mathrm{MeV}\right)$ and transverse momentum broadening $\left(\left\langle\Delta k_{T}^{2}\right\rangle \simeq 0.2-0.3 \mathrm{GeV}^{2}\right)$. Predictions for photon spectra to be measured at higher colliding energies (RHIC and LHC) have also been made, suggesting transverse-momentum windows around $3 \mathrm{GeV}$ as promising to track QGP radiation. 


\section{Acknowledgments}

We are grateful to K. L. Haglin for useful communications in connection with his parallel investigation of reactions with strangeness, discussed in Section IIB of this article. We are happy to acknowledge useful discussions with T. C. Awes and D. K. Srivastava. The work of ST and CG was supported in part by the Natural Sciences and Engineering Research Council of Canada, and in part by the Fonds Nature et Technologies of Quebec.

[1] See, for example, Proceedings of the 16. International Conference on Ultrarelativistic Nucleus-Nucleus Collisions, Quark Matter 2002, Nantes, France, 18-24 July 2002, Nucl. Phys. A715 (2003).

[2] L.D. McLerran and T. Toimela, Phys. Rev. D31 1545 (1985); H.A. Weldon, Phys. Rev. D42, 2384 (1990); C. Gale and J.I. Kapusta, Nucl. Phys. B357, 65 (1991).

[3] T. Peitzmann and M. Thoma, Phys. Rep. 364, 175 (2002); C. Gale and K.L. Haglin, arXiv:hep-ph/0306098

[4] P. Aurenche et al., Eur. Phys. J. C9, 107 (1999).

[5] J.W. Cronin, H.J. Frisch, M.J. Shochet, J.P. Boymond, R. Mermod, P.A. Piroue, and R.L. Summer, Phys. Rev. D 11, 3105 (1975).

[6] J. Owens, Rev. Mod. Phys. 59, 465 (1987);

[7] K. Eskola, V. Kolhinen, and V. Ruuskanen, Nucl. Phys. B535, 351 (1998).

[8] A. Dumitru et al., Phys. Rev. C51, 2166 (1995).

[9] D.K. Srivastava and B. Sinha, Phys. Rev. C64, 034902 (2001).

[10] P. Huovinen, P.V. Ruuskanen and S.S. Räsänen, Phys. Lett. B535, 109 (2002).

[11] M.-A. Halasz, J.V. Steele, G.Q. Li and G.E. Brown, Phys. Rev. C58, 365 (1998).

[12] D.K. Srivastava and K. Geiger, Phys. Rev. C58, 1734 (1998).

[13] P. Huovinen, M. Belkacem, P. J. Ellis, and J. I. Kapusta, Phys. Rev. C 66, 014903 (2002).

[14] R. Rapp and J. Wambach, Proc. of the 2. KEK-Tanashi International Symposium on Hadron and Nuclear Physics with Electromagnetic Probes, (Tanashi, Tokyo, 25.-27.10.99), edited by K. Maruyama and H. Okuno, ELSEVIER (Amsterdam, 2000) p. 21, arXiv:nucl-th/0001014.

[15] K. Gallmeister, B. Kämpfer and O.P. Pavlenko, Phys. Rev. C62, 057901 (2000).

[16] R. Rapp and J. Wambach, Adv. Nucl. Phys. 25 (2000) 1.

[17] CERES/NA45 Collaboration (D. Adamova et al.), Phys. Rev. Lett. 91, 042301 (2003) arXiv:nucl-ex/0209024.

[18] J.J. Sakurai, Currents and Mesons, (University of Chicago Press, Chicago, 1969); F. Klingl, N. Kaiser, and W. Weise, Z. Phys. A 356, 193 (1996); H.B. O'Connell, B.C. Pearce, A.W. Thomas, and A.G. Williams, Prog. Part. Nucl. Phys. 39, 201 (1997).

[19] H. Gomm, Ö. Kaymakcalan, and J. Schechter, Phys. Rev. D30, 2345 (1984).

[20] C. Song, Phys. Rev. C47, 2861 (1993).

[21] S. Gao and C. Gale, Phys. Rev. C57, 254 (1998).

[22] Particle Data Group, Phys. Rev. D66, 010001 (2002).

[23] C. Song and G. Fai, Phys. Rev. C58, 1689 (1998).

[24] Reaction (iii) in part 1 and reaction (ii) in part 2 of the Appendix A in [20] contain a Ward identity-violating component.

[25] J. Kapusta, P. Lichard and D. Seibert, Phys. Rev. D44, 2774 (1991) [erratum-ibid. D47, 4171 (1991)].

[26] R. Rapp and C. Gale, Phys. Rev. C60, 024903 (1999).

[27] K. Haglin, Phys. Rev. C 50, 1688 (1994).

[28] C.-H. Lee, H. Yamagishi, and I. Zahed, Phys. Rev. C 58, 2899 (1998).

[29] R. Rapp, talk at the 3. plenary meeting of the CERN workshop on Hard Probes in Heavy-Ion Collisions at the LHC, CERN (Geneva), Oct. 7-11, 2002; http://wwwth.cern.ch/lhcworkshop/plenary_oct02.html

[30] R. Rapp, Proc. of 4. ICPAQGP (Jaipur, India, Nov. 26-30, 2001), Pramana 60, 675 (2003) arXiv:hep-ph/0201101.

[31] CERES/NA45 Collaboration (G. Agakichiev et al.), Phys. Rev. Lett. 75, 1272 (1995);

Phys. Lett. B422, 405 (1998); Nucl. Phys. A661, 23 (1999).

[32] J.V. Steele, H. Yamagishi and I. Zahed, Phys. Rev. D56, 5605 (1997).

[33] G.Q. Li and G.E. Brown, Nucl. Phys. A632, 153 (1998).

[34] J. Alam, P. Roy and S. Sarkar, arXiv:nucl-th/0304056

[35] R. Rapp, G. Chanfray and J. Wambach, Nucl. Phys. A617, 472 (1997).

[36] R. Rapp and J. Wambach, Eur. Phys. J. A6, 415 (1999).

[37] R. Rapp, M. Urban, M. Buballa and J. Wambach, Phys. Lett. B417, 1 (1998).

[38] A. Majumder and C. Gale, Phys. Rev. C65, 055203 (2002).

[39] J.V. Steele and I. Zahed, Phys. Rev. D60, 037502 (1999).

[40] P. Arnold, G.D. Moore and L.G. Yaffe, JHEP 0112, 9 (2001).

[41] F. Karsch, E. Laermann, P. Petreczky, S. Stickan and I. Wetzorke, Phys. Lett. B530, 147 (2002).

[42] R. Rapp and E.V. Shurayk, Phys. Lett. B473, 13 (2000).

[43] P. Braun-Munzinger, I. Heppe and J. Stachel, Phys. Lett. B465, 15 (1999); 
P. Braun-Munzinger, D. Magestro, J. Stachel and K. Redlich, Phys. Lett. B518, 41 (2001).

[44] R. Rapp, Phys. Rev. C66, 017901 (2002).

[45] D.K. Srivastava, Eur. Phys. J. C22, 129 (2001).

[46] M. McLaughlin et al.(E629 Collaboration), Phys. Rev. Lett. 31, 971 (1983).

[47] J. Badier et al.(NA3 Collaboration), Z. Phys. C31, 341 (1986).

[48] WA98 Collaboration (M.M. Aggarwal et al.), Phys. Rev. Lett. 85, 3593 (2000).

[49] S.A. Bass, B. Müller, and D.K. Srivastava, Phys. Rev. Lett. 90, 082301 (2003).

[50] NA50 Collaboration (M.C. Abreu et al.), Eur. Phys. J. C14, 443 (2000);

NA50 Collaboration (L. Capelli et al.), Nucl. Phys. A698, 539 (2002).

[51] I. Kvasnikova, C. Gale, and D.K. Srivastava, Phys. Rev. C65, 064903 (2002).

[52] WA80 Collaboration (R. Albrecht et al.), Phys. Rev. Lett. 76, 3506 (1996).

[53] F. Karsch, E. Laermann and A. Peikert, Phys. Lett. B478, 447 (2000).

[54] T. Renk, Phys. Rev. C 67, 064901 (2003) arXiv:hep-ph/0301133.

[55] A. Dumitru, L. Frankfurt, L. Gerland, H. Stöcker, and M. Strickman, Phys. Rev. C64, 054909 (2001).

[56] G. Papp, P. Lévai, and G. Fai, Phys. Rev. C61, 021902(R) (1999).

[57] WA98 Collaboration (M.M. Aggarwal et al.), Eur. Phys. J. C23, 225 (2002).

[58] L. Grandchamp and R. Rapp, Nucl. Phys. A709, 415 (2002);

L. Grandchamp, R. Rapp and G.E. Brown, arXiv:hep-ph/0306077

[59] NA50 Collaboration (M.C. Abreu et al.), Phys. Lett. B477, 28 (2000);

NA50 Collaboration (L. Ramello et al.), Nucl. Phys. A715, 243 (2003).

[60] BRAHMS Collaboration (I.G. Bearden et al.), Phys. Rev. Lett. 88, 202301 (2002).

[61] PHOBOS Collaboration (B.B. Back et al.), Phys. Rev. C65, 061901 (2002).

[62] I. Tserruya, Proc. of 4. ICPAQGP (Jaipur, India, Nov. 26-30, 2001), Pramana 60, 577 (2003) arXiv:nucl-ex/0204012.

[63] R. Rapp, Phys. Rev. C63, 054907 (2001).

[64] PHENIX Collaboration (S.S. Adler et al.), arXiv:nucl-ex/0306021

[65] STAR Collaboration (J. Adams et al.), arXiv:nucl-ex/0306024

[66] PHOBOS Collaboration (B.B. Back et al.), arXiv:nucl-ex/0306025

[67] P.F. Kolb and U. Heinz, arXiv:nucl-th/0305084

[68] J. Cleymans, K. Redlich and D.K. Srivastava, Phys. Lett. B420, 261 (1998). 


\section{APPENDIX A: PARAMETERISATIONS}

The photon emission rates have been calculated from the Lagrangian describe in Sec. II and by the VMD interaction

$$
\mathcal{L}_{e m}=-C m_{\rho}^{2} A^{\mu} \rho_{\mu}^{0}
$$

where $A^{\mu}$ is the photon field and $C$ is a constant adjusted by the experimental decay $\rho^{0} \rightarrow e^{+} e^{-}$, which gives $\mathrm{C}=0.059$. In order to respect the Ward Identity in a direct way, we multiply each Feynman amplitude by the square of the averaged space-like form factor of Eq. (10). Time-like form factors have been defined to be normalised to one for onshell decays. We quote below parametrisations which include the axial meson $a_{1}$ as exchange particle for non-strange initial states. In the following, the photon energy $(E)$ and the temperature $(T)$ are both in GeV. Parameterisations for $K^{*} \rightarrow K+\pi+\gamma$ and $K+K \rightarrow \rho+\gamma$ do not appear because their rates have been found to be negligible.

$$
\begin{aligned}
& E \frac{d R_{\pi+\rho \rightarrow \pi+\gamma}}{d^{3} p}=F^{4}(E) T^{2.8} \exp \left(\frac{-\left(1.461 T^{2.3094}+0.727\right)}{(2 T E)^{0.86}}+\left(0.566 T^{1.4094}-0.9957\right) \frac{E}{T}\right)\left(\mathrm{fm}^{-4} \mathrm{GeV}^{-2}\right) \\
& E \frac{d R_{\pi+\pi \rightarrow \rho+\gamma}}{d^{3} p}=F^{4}(E) \frac{1}{T^{5}} \exp \left(-\left(9.314 T^{-0.584}-5.328\right)(2 T E)^{0.088}+\left(0.3189 T^{0.721}-0.8998\right) \frac{E}{T}\right) \\
& E \frac{d R_{\rho \rightarrow \pi+\pi+\gamma}}{d^{3} p}=F^{4}(E) \frac{1}{T^{2}} \exp \left(-\frac{\left(-35.459 T^{1.126}+18.827\right)}{(2 T E)^{\left(-1.44 T^{0.142}+0.9996\right)}}-1.21 \frac{E}{T}\right) \\
& E \frac{d R_{\pi+K^{*} \rightarrow K+\gamma}}{d^{3} p}=F^{4}(E) T^{3.75} \exp \left(-\frac{0.35}{(2 T E)^{1.05}}+\left(2.3894 T^{0.03435}-3.222\right) \frac{E}{T}\right) \\
& E \frac{d R_{\pi+K \rightarrow K^{*}+\gamma}}{d^{3} p}=F^{4}(E) \frac{1}{T^{3}} \exp \left(-\left(5.4018 T^{-0.6864}-1.51\right)(2 T E)^{0.07}-0.91 \frac{E}{T}\right) \\
& E \frac{d R_{\rho+K \rightarrow K+\gamma}}{d^{3} p}=F^{4}(E) T^{3.5} \exp \left(-\frac{\left(0.9386 T^{1.551}+0.634\right)}{(2 T E)^{1.01}}+\left(0.568 T^{0.5397}-1.164\right) \frac{E}{T}\right) \\
& E \frac{d R_{K^{*}+K \rightarrow \pi+\gamma}}{d^{3} p}=F^{4}(E) T^{3.7} \exp \left(\frac{-\left(6.096 T^{1.889}+1.0299\right)}{(2 T E)^{\left(-1.613 T^{2.162}+0.975\right)}}-0.96 \frac{E}{T}\right)
\end{aligned}
$$

$F(E)$ is the form factor, cf. Sec. ПB 